\title{
Development of GPS Aided Inertial Navigation System for High Speed Flight Demonstrator
}

\author{
Masatoshi HarigaE* ${ }^{*}$ Hiroshi Tomita* and Takeshi NishizawA**
}

\begin{abstract}
NAL and NASDA develop High Speed Flight Demonstrators (HSFDs) to examine an automatic take-off and landing technology (Phase 1) and to measure the transonic aerodynamic characteristics of a reusable space plane for getting the reference data of a CFD (Computed Fluid Dynamics) technology (Phase 2). For HSFD navigation, we develop a GPS aided inertial navigation system (GAINS) whose distinctive feature is using the carrier-phase DGPS (CDGPS)/INS hybrid navigation technology. This paper describes the design of GAINS. We show its accuracy, integrity and continuity performances that are analyzed by numerical simulations using the covariance analysis technique. Ground and flight tests are also carried out to confirm the performance of the GAINS flight model. Both results prove that GAINS meets the requirements of the navigation performance under severe flight conditions of HSFD.
\end{abstract}

Key Words: GPS/INS, precision approach and landing, integrity, flight test

\section{INTRODUCTION}

NAL and NASDA are now developing experimental vehicles, named High Speed Flight Demonstrator (HSFD), whose first flight is planned in 2002. HSFDs are fourth test vehicles of reusable space plane HOPE (H-II Orbiting Plane) and their missions are divided into two phases. In the phase 1 mission to be carried out at Christmas Island of the Republic of Kiribati, we examine the performance of the onboard navigation, guidance and control system from the TAEM (Total Energy Management) phase to the precision approach and landing phase. The vehicle equipped with a jet engine automatically takes off and lands several times during the experiments. In the phase 2 mission planned at Esrange of the Kingdom of Sweden in cooperation with CNES, we measure the aerodynamic characteristics (relations between the vehicle configuration and aerodynamic forces) of the small-scaled HOPE vehicle under the transonic speed (Mach 0.8 1.2).

A navigation system of HSFD has to meet the high-level requirements in its accuracy, integrity and continuity. The conventional DGPS and Real-time Kinematic GPS (RTKGPS) navigation systems are not enough to satisfy the requirements at the precision approach and landing phase on account of high dynamic motions of HSFD. This paper describes the development of a GPS aided inertial navigation system for HSFD whose distinctive feature is using

* National Aerospace Laboratory of Japan (NAL)

** National Space Development Agency of Japan (NASDA) (Received May 13, 2002)

(Revised November 11, 2002) the carrier-phase DGPS (CDGPS)/INS hybrid navigation technology. CDGPS/INS navigation is a novel approach to realize high navigation accuracy without lack of good integrity and continuity performance. Because the ambiguity of the carrier phase is resolved with the aid of INS information, CDGPS/INS navigation can avoid the quick degradation of navigation performance when the GPS signal lock-loss and re-acquisition occur under the high dynamic motions.

This paper describes the design of GAINS. Numerical simulations using the covariance analysis technique show the accuracy, integrity and continuity performances of GAINS in HSFD missions. Ground and flight tests are also carried out to confirm the performance of the GAINS flight model and the correctness of the theoretical analysis. Both results prove that GAINS meets the requirements of the navigation performance under severe flight conditions of HSFD.

\section{HSFD MISSION OVERVIEW AND NAVIGATION REQUIREMENT ${ }^{1)}$}

Fig. 1 shows the HSFD phase 1 mission scenario. HSFD autonomously takes off the runway and demonstrates the capability of the onboard GN\&C system and the data link system during a free flight. There are two trajectories one of which simulates the HOPE trajectory from TAEM to approach and landing. This trajectory is selected to prove the GN\&C performance under HOPE similar flight conditions. The other includes $50 \mathrm{~km}$ away flight from the ground stations where we check the data link performance. 


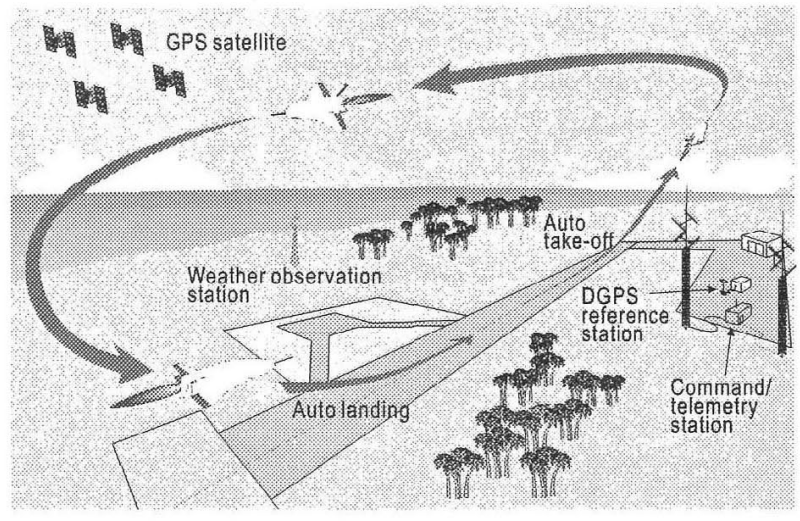

Fig. 1 HSFD phase 1 mission scenario

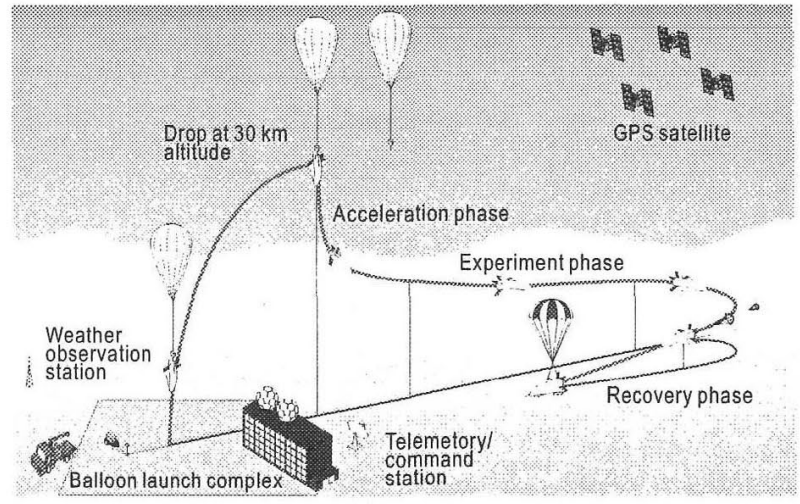

Fig. 2 HSFD phase 2 mission scenario

Table 1 Requirement on navigation accuracy $(3 \sigma)$

\begin{tabular}{|c|c|c|c|}
\hline Mission Phase & Position (m) & Velocity $(\mathrm{m} / \mathrm{s})$ & Attitude (deg) \\
\hline Take-off & L 10.0 & & $\mathrm{R} / \mathrm{P} 0.2$ \\
\hline \multirow{2}{*}{ Cruise $(>$ h $3000 \mathrm{~m})$} & L 350.0 & & \\
\hline & V 490.0 & & \\
\hline \multirow[t]{2}{*}{1 Cruise $(<\mathrm{h} 3000 \mathrm{~m})$} & L 10.0 & & \\
\hline & V 10.0 & & \\
\hline \multirow[t]{2}{*}{ Approach/Landing } & L 10.0 & & $\mathrm{R} / \mathrm{P} 0.2$ \\
\hline & V 1.0 & V 1.0 & Y 0.5 \\
\hline Taxing & L 10.0 & & \\
\hline \multirow[t]{2}{*}{$\begin{array}{ll}2 & \text { Recovery }\end{array}$} & L 24.0 & & \\
\hline & V 70.0 & & \\
\hline
\end{tabular}

Note: h $\cdots$ Height, L $\cdots$ Lateral, V $\cdots$ Vertical, R/P $\cdots$ Roll/Pitch, Y $\cdots$ Yaw

Blank cell means not available.

Fig. 2 shows the HSFD phase 2 mission scenario. A balloon launches HSFD (with no propulsion system) that reaches $30 \mathrm{~km}$ altitude after 2 or 3 hours flight. Then HSFD is dropped at that altitude and then accelerates up to the transonic speed. During the steady speed period to be realized by appropriate maneuvers, we measure aerodynamic characteristics. After the measurement mission, HSFD selects the suitable trajectory to a recovery point and glides autonomously. At the recovery point, HSFD opens parachutes and air bags for safety landing.
We plan six flights with three steady speed (Mach 0.8, 1.05 and 1.2) phases.

Primary navigation requirement is set on the accuracy of the onboard navigation system. Table 1 shows the requirement for HSFD phase 1 and 2. Most critical requirement exists in the vertical position error for the approach and landing phase. The flight experiments showed that the vertical position error of the conventional DGPS/INS hybrid navigation system was $2 \sim 3 \mathrm{~m}$ $(3 \sigma)^{2)}$ that did not meet the requirement. To satisfy this requirement, GAINS has a carrier-phase DGPS/INS (CDGPS/INS) hybrid navigation mode to be described later in detail. Besides accuracy, integrity and continuity requirement is also important to develop a navigation system. The HSFD missions do not determine the requirement on integrity and continuity explicitly. Then we refer LAAS specifications proposed by RTCA SC-159 ${ }^{3), 4)}$ in the analysis. Availability that is a probability meeting the three performance requirements simultaneously should be more than $99 \%$ in the HSFD missions.

\section{GAINS CONFIGURATION}

GAINS is a differential GPS aided inertial navigation system. It consists of onboard equipment and a ground station as shown in Fig. 3. In the onboard equipment, date of an IMU (ring laser gyro and accelerometer) are integrated with date of a GPS receiver in a processing unit. A telemetry receiver gets raw observation data of a reference GPS receiver via RTCM-104 type 18 and 19 message. As described in the next section, GAINS performs CDGPS/INS hybrid navigation where a carrier phase smoothes navigation data so that multi GPS receivers are not necessary at the ground station to achieve sub-meter positioning accuracy. In the ground station, there is a monitor system to check the quality of the reference GPS receiver data and a transmilter. To check the quality of the DGPS data, DGPS stand-alone navigation is carried out in a processor of the monitor system.

\section{GAINS NAVIGATION ALGORITHM ${ }^{5)}$}

GAINS adopts a tightly coupled GPS/INS hybrid navigation algorithm that corrects the IMU errors as well as the INS navigation data by using the single difference GPS data. It has various navigation modes such as INS, GPS/INS, DGPS/INS and CDGPS/INS. The INS mode enables GAINS to provide navigation data even though GPS signal is lost. This function improves the continuity performance. In the GPS/INS mode, GAINS carries out calibration of the INS errors and avoids divergence of 
navigation errors with GPS data. The DGPS/INS and CDGPS/INS mode assure most accurate navigation data under the condition that both GPS and a telemetry link are available. GAINS changes its navigation mode autonomously, whose mode transition policy is to provide most accurate data that are available according to GPS and the telemetry conditions.

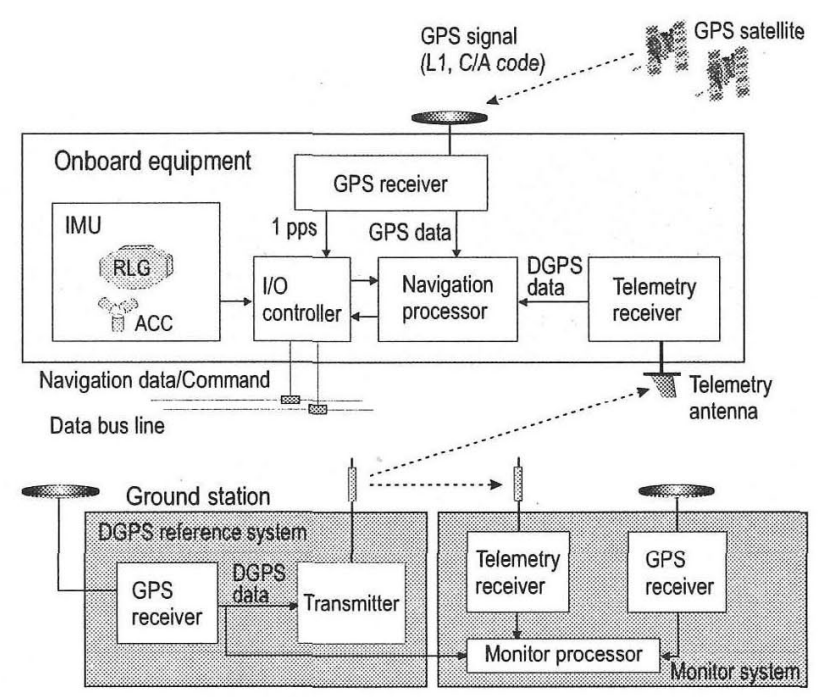

Fig. 3 GAINS block diagram

The CDGPS/INS mode is prepared to be able to satisfy the most critical requirement on vertical navigation errors during the approach and landing phase. The navigation algorithm of this mode is different from the conventional Kinematic GPS/INS hybrid navigation that also uses carrier phase data. In this mode, the navigation algorithm does not include ambiguity search and fix mechanism like Kinematic GPS, but uses a Kalman filter that estimates the ambiguity as one of GPS error sources. GAINS first sets an initial value of the ambiguity for each carrier phase measurement, which is calculated from a DGPS/INS positioning result (It means the CDGPS/INS mode is transited from the DGPS/INS mode). Then the Kalman filter calibrates the ambiguity along with other navigation errors. In the Kalman filter, the following error dynamics and measurement equation are used.

\section{1 Error dynamics model}

$$
\dot{\boldsymbol{x}}=\boldsymbol{F} \boldsymbol{x}+\boldsymbol{w}
$$

$$
\boldsymbol{x}=\left(\boldsymbol{\delta} r, \boldsymbol{\delta} \boldsymbol{v}, \boldsymbol{\delta} e, \boldsymbol{b}_{a c c}, \boldsymbol{b}_{g y r o}, \boldsymbol{b}_{c l k}, \boldsymbol{n}\right)^{T}
$$

$$
\boldsymbol{F}=\left[\begin{array}{ccccccc}
\boldsymbol{F}_{r r} & \boldsymbol{F}_{r v} & 0 & 0 & 0 & 0 & 0 \\
\boldsymbol{F}_{v r} & \boldsymbol{F}_{v v} & \boldsymbol{F}_{v e} & \boldsymbol{F}_{v b} & 0 & 0 & 0 \\
\boldsymbol{F}_{e r} & \boldsymbol{F}_{e v} & \boldsymbol{F}_{e e} & 0 & \boldsymbol{F}_{e b} & 0 & 0 \\
0 & 0 & 0 & \boldsymbol{F}_{b a} & 0 & 0 & 0 \\
0 & 0 & 0 & 0 & \boldsymbol{F}_{b g} & 0 & 0 \\
0 & 0 & 0 & 0 & 0 & \boldsymbol{F}_{b c} & 0 \\
0 & 0 & 0 & 0 & 0 & 0 & \boldsymbol{F}_{n}
\end{array}\right]
$$$$
\boldsymbol{w}=\left(0,0,0, \boldsymbol{w}_{b a}, \boldsymbol{w}_{b g}, \boldsymbol{w}_{b c}, \boldsymbol{w}_{n}\right)^{T}
$$

where $\delta r, \delta v, \delta e$ are the position, velocity and attitude error vectors respectively; $\boldsymbol{b}_{a c c}$ and $\boldsymbol{b}_{\text {gyro }}$ are the accelerometer bias and gyro bias vectors; $\boldsymbol{b}_{c l k}$ is the GPS receiver clock error vector; $\boldsymbol{n}$ is the ambiguity vector. The ambiguity is treated as a float variable. Except the ambiguity vector $n$, the error dynamics model is same to that of the conventional DGPS/INS hybrid navigation. The reference frame of the system matrix $\boldsymbol{F}$ is the navigation frame. The dynamics of the ambiguity is modeled as the first-order Markov process whose time constant is dependent on the GPS atmospheric error.

\section{2 Measurement equation model}

For INS calibration, GAINS uses both the pseudorange and the carrier phase in the CDGPS/INS mode. For the pseudorange, the following measurement model is used.

$$
\begin{array}{r}
\Delta \rho=\left|\boldsymbol{r}-\boldsymbol{r}_{s v}\right|-\left|\boldsymbol{r}_{b}-\boldsymbol{r}_{s v}\right|+\Delta b_{s a g} \\
+\Delta b_{c l k}+w_{\rho}
\end{array}
$$

where $\Delta \rho$ is the single difference of the pseudorange; $\boldsymbol{r}, \boldsymbol{r}_{b}, \boldsymbol{r}_{s v}$ are the position vectors of the user, the DGPS reference receiver and the GPS satellite respectively; $\Delta b_{\text {sag }}$ is the single difference of the sagnac effect; $\Delta b_{c l k}$ is the single difference of the receiver clock error; $w_{\rho}$ represents the measurement noise. We suppose no atmospheric error remained in the single difference.

For the carrier phase, we add the ambiguity to the measurement equation as follows.

$$
\begin{array}{r}
\Delta \phi=\frac{f_{L 1}}{c}\left\{\left|r-r_{s v}\right|-\right. \\
\left.+\Delta r_{b}-r_{s v} \mid+\Delta b_{s a g}\right\}+n+w_{\phi}
\end{array}
$$

where $f_{L 1}$ is the center frequency of GPS L1 band; $c$ is the light speed; $n$ is the ambiguity ${ }^{6)} ; w_{\phi}$ is the measurement noise of the carrier phase. The difference between the CDGPS/INS mode and the DGPS/INS mode is whether the carrier phase measurement is used or not. Then the Kalman filter does not estimate the ambiguity $n$ in the DGPS/INS mode.

To check the integrity of navigation, GAINS uses the 
Table 2 State vector models of GPS and INS errors in covariance analysis

\begin{tabular}{|c|c|c|c|c|}
\hline Error sources & $\begin{array}{l}\text { Number } \\
\text { of state }\end{array}$ & Magnitude $(1 \sigma)$ & Dynamics model & $\begin{array}{l}\text { DGPS effective } \\
\text { figure }\end{array}$ \\
\hline Ambiguity & 12 & $0.5 \mathrm{~m}$ (initial value) & Steady bias & 1.0 \\
\hline Clock error & 4 & $10^{-10}($ at $1 \mathrm{sec})$ & $\begin{array}{l}\text { Bias, frequency random walk, frequency } \\
\text { flicker noise }\end{array}$ & 1.0 \\
\hline Pseudorange bias & 12 & depend on the analysis & $\begin{array}{l}\text { Steady bias } \\
\text { (used only in the integrity analysis) }\end{array}$ & 1.0 \\
\hline Carrier phase bias & 12 & depend on the analysis & $\begin{array}{l}\text { Steady bias } \\
\text { (used only in the integrity analysis) }\end{array}$ & 1.0 \\
\hline Ionospheric delay & 12 & $1.5 \mathrm{~m} \sim 4.0 \mathrm{~m}$ & $\begin{array}{l}\text { Steady bias whose magnitude yields to the } \\
\text { Bent 8-parameter model }\end{array}$ & $0.0011 l$ \\
\hline Tropospheric delay & 1 & $2.5 \mathrm{~m} \sim 12.0 \mathrm{~m}$ & $\begin{array}{l}\text { Steady bias whose magnitude yields to the } \\
\text { Saastamoinen model with CfA }-2.2 \text { mapping }\end{array}$ & 0.005 \\
\hline Multipath & 12 & $\begin{array}{l}0.25 \mathrm{~m} \text { (pseudorange) } \\
0.5 \mathrm{~cm} \text { (carrier phase) }\end{array}$ & $\begin{array}{l}\text { First-order Markov process with } 50 \mathrm{sec} \\
\text { time constant }\end{array}$ & 1.0 \\
\hline Receiver noise & - & $\begin{array}{c}0.5 \mathrm{~m} \text { (pseudorange) } \\
0.5 \mathrm{~cm} \text { (carrier phase) }\end{array}$ & White noise & 1.0 \\
\hline Initial position error & 3 & $100 \mathrm{~m}$ & $\begin{array}{l}\text { Derivative of position error is velocity } \\
\text { error }\end{array}$ & - \\
\hline Initial velocity error & 3 & $1 \mathrm{~m} / \mathrm{sec}$ & $\begin{array}{l}\text { Derivative of velocity error is acceleration } \\
\text { error }\end{array}$ & - \\
\hline Initial attitude error & 3 & $0.01 \mathrm{deg}$ & $\begin{array}{l}\text { Derivative of attitude error is angular } \\
\text { rate error }\end{array}$ & - \\
\hline IMU bias & 6 & $\begin{array}{c}80 \mu \mathrm{G} \text { (acc) } \\
0.005 \mathrm{deg} / \mathrm{h} \text { (gyro) }\end{array}$ & $\begin{array}{l}\text { First-order Markov process with } 600 \mathrm{sec} \\
\text { time constant }\end{array}$ & - \\
\hline IMU scale factor & 6 & $\begin{array}{l}130 \text { PPM (acc) } \\
10 \text { PPM (gyro) }\end{array}$ & Steady bias & - \\
\hline IMU misalignment & 12 & $\begin{array}{l}10 \operatorname{arcsec}(\text { acc }) \\
2 \operatorname{arcsec} \text { (gyro) }\end{array}$ & Steady bias & - \\
\hline
\end{tabular}

Note: $\quad l \cdots$ distance between the onboard equipment and the ground station $(\mathrm{km})$ acc $\cdots$ accelerometer

measurement residuals in the Kalman filter. The carrier phase measurement residual is especially monitored to find the cycle slip in the carrier phase. When the cycle slip is detected, the initial value of the ambiguity is again calculated from the CDGPS/INS position at that time. If the INS calibration is sufficiently carried out, the recalculated ambiguity is close to the correct value so that we can avoid the much degradation or jump of navigation accuracy without time loss for ambiguity search. The detailed description of the integrity check algorithm will be shown later.

\section{THEORETICAL ANALYSIS OF NAVIGATION PERFORMANCE}

\subsection{Accuracy}

\subsubsection{Analysis Method}

Using the following algorithm that yields to the covariance analysis technique, we evaluate the accuracy of GAINS. Let us define the navigation error by the following form.

$$
\begin{aligned}
\tilde{\boldsymbol{X}} & =\hat{\boldsymbol{X}}-\boldsymbol{X} \\
\hat{\boldsymbol{X}} & =(\hat{\boldsymbol{x}}, O)^{T}
\end{aligned}
$$

$$
\boldsymbol{X}=\left(\boldsymbol{x}, \boldsymbol{x}_{e r r}\right)^{T}
$$

where $\boldsymbol{x}$ denotes the state vector of the Kalman filter shown in equation (1) and $\boldsymbol{x}_{e r r}$ is the state vector that is not included in the Kalman filter but affects the navigation accuracy such as the atmospheric error, IMU misalignments, etc.Then we suppose the vector $\boldsymbol{X}$ and their dynamics as shown in Table 2. In this table, we introduce a DGPS effective figure that reflects how much GPS error sources remain in a single difference.

The covariance of the navigation error after time update is presented as follows.

$$
\begin{aligned}
\boldsymbol{P}_{k}^{-} & =E\left[\widetilde{\boldsymbol{X}}_{k}^{-}\left(\tilde{\boldsymbol{X}}_{k}^{-}\right)^{T}\right] \\
& =\boldsymbol{\Phi}_{k, k-1} \boldsymbol{P}_{k-1}^{+} \boldsymbol{\Phi}_{k, k-1}^{T}+\boldsymbol{Q}_{k, k-1}
\end{aligned}
$$

where $\boldsymbol{\Phi}$ is the transition matrix; $\boldsymbol{Q}$ is the covariance matrix of the process noise. After measurement update, the covariance matrix $\boldsymbol{P}$ becomes,

$$
\begin{aligned}
\boldsymbol{P}_{k}^{+} & =\left(\boldsymbol{I}-\boldsymbol{W}^{T} \boldsymbol{k}_{k} \boldsymbol{h}_{k}^{T} \boldsymbol{W}\right) \boldsymbol{P}_{k}^{-}\left(\boldsymbol{I}-\boldsymbol{W}^{T} \boldsymbol{k}_{k} \boldsymbol{h}_{k}^{T} \boldsymbol{W}\right)^{T}(6) \\
& +\boldsymbol{W}^{T} \boldsymbol{k}_{k} \boldsymbol{r}_{k} \boldsymbol{k}_{k}^{T} \boldsymbol{W}
\end{aligned}
$$

where $k, h, r$ are the onboard Kalman gain, the measurement matrix and the covariance of the measurement noise respectively. The matrix $W$ is for changing the di- 
Table 3 Nominal conditions supposed for the analysis

\begin{tabular}{|c|c|c|}
\hline & Item & Condition \\
\hline \multirow[t]{2}{*}{$\begin{array}{l}\text { Space } \\
\text { segment }\end{array}$} & Ephemeris & $\begin{array}{l}28 \text { satellite, collected on January, } \\
2000 \text { for Phase } 1 \text { and on May, } 2000 \\
\text { for Phase } 2\end{array}$ \\
\hline & Mask angle & $10 \mathrm{deg}$ in elevation \\
\hline \multirow{3}{*}{$\begin{array}{l}\text { User } \\
\text { segment }\end{array}$} & Receiver & $\begin{array}{l}\text { According to specifications of } \\
\text { Ashtech G12 }\end{array}$ \\
\hline & Antenna & $\begin{array}{l}\text { Sufficient gain available above } 10 \\
\text { deg in its elevation }\end{array}$ \\
\hline & IMU & $\begin{array}{l}\text { According to specifications of } \\
\text { Kearfott T24 }\end{array}$ \\
\hline \multirow{3}{*}{$\begin{array}{l}\text { Phase } 1 \\
\text { mission }\end{array}$} & Date & $\begin{array}{l}\text { Take off on January } 13 \text { at Christ- } \\
\text { mas Island }\end{array}$ \\
\hline & Trajectory & $\begin{array}{l}\text { HOPE simulation and } 50 \mathrm{~km} \text { away } \\
\text { flight trajectory }\end{array}$ \\
\hline & Antenna & $\begin{array}{l}1 \text { GPS antenna located at the top } \\
\text { of HSFD body }\end{array}$ \\
\hline \multirow[b]{2}{*}{$\begin{array}{l}\text { Phase } 2 \\
\text { mission }\end{array}$} & Date & Launch on May 11 at Esrange \\
\hline & $\begin{array}{l}\text { Trajectory } \\
\text { Antenna }\end{array}$ & $\begin{array}{l}30 \mathrm{~km} \text { altitude trajectory } \\
1 \mathrm{GPS} \text { antenna is located at the } \\
\text { gondola of the balloon and the } \\
\text { other is located at the top of HSFD } \\
\text { body, which are switched at the re- } \\
\text { lease time }\end{array}$ \\
\hline
\end{tabular}

Table 4 Analysis result on navigation accuracy $(3 \sigma)$ of GAINS for HSFD missions

\begin{tabular}{l|c|c|c}
\hline \multirow{2}{*}{ Trajectory } & \multicolumn{2}{|c|}{ Phase $1^{* 1}$} & Phase $2^{* 2}$ \\
\cline { 2 - 4 } & HOPE & $50 \mathrm{~km}$ away & $30 \mathrm{~km}$ altitude \\
\hline \multirow{2}{*}{ Position } & $\mathrm{L} 0.4 \mathrm{~m}$ & $\mathrm{~L} 0.2 \mathrm{~m}$ & $\mathrm{~L} 14.0 \mathrm{~m}$ \\
& $\mathrm{~V} 1.6 \mathrm{~m}$ & $\mathrm{~V} 0.7 \mathrm{~m}$ & $\mathrm{~V} 40.0 \mathrm{~m}$ \\
\hline \multirow{2}{*}{ Velocity } & $\mathrm{L} 1.4 \mathrm{~cm} / \mathrm{s}$ & $\mathrm{L} 0.7 \mathrm{~cm} / \mathrm{s}$ & $\mathrm{L} 24.0 \mathrm{~cm} / \mathrm{s}$ \\
& V $4.2 \mathrm{~cm} / \mathrm{s}$ & V $1.7 \mathrm{~cm} / \mathrm{s}$ & $\mathrm{L} 25.0 \mathrm{~cm} / \mathrm{s}$ \\
\hline \multirow{2}{*}{ Attitude } & R $/ \mathrm{P} 0.003 \mathrm{deg}$ & $\mathrm{R} / \mathrm{P} 0.004 \mathrm{deg}$ & $\mathrm{R} / \mathrm{P} 0.005 \mathrm{deg}$ \\
& Y $0.010 \mathrm{deg}$ & Y $0.008 \mathrm{deg}$ & Y $0.145 \mathrm{deg}$ \\
\hline Worst case & $16: 20$ take-off & $09: 40$ take-off & $09: 00$ launch \\
\hline
\end{tabular}

Note: $*^{*}$ CDGPS/INS mode, $*^{2}$ GPS/INS mode

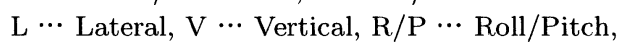
Y $\cdots$ Yaw

mension from $\boldsymbol{X}$ to $\boldsymbol{x}$. The covariance matrix $\boldsymbol{P}_{R}^{+}$denotes the accuracy of GAINS.

\subsubsection{Results of Analysis in HSFD Mission}

For the analysis, we suppose nominal conditions of GPS space segment, user segment and the HSFD Phase 1 and 2 missions as shown in Table 3. Fig. 4 shows the trajectories for the phase 1 and 2 described in Table 3 . There are three trajectories to be analyzed. To carry out the worst-case analysis, we look for the time when the GPS satellite visibility is poorest. In the HSFD phase 1 mission, we select the worst GDOP case, averaged for $200 \mathrm{sec}$ before landing, because the most critical requirement is set on the approach and landing phase. In the HSFD phase 2 mission, we chose the case when GDOP at the
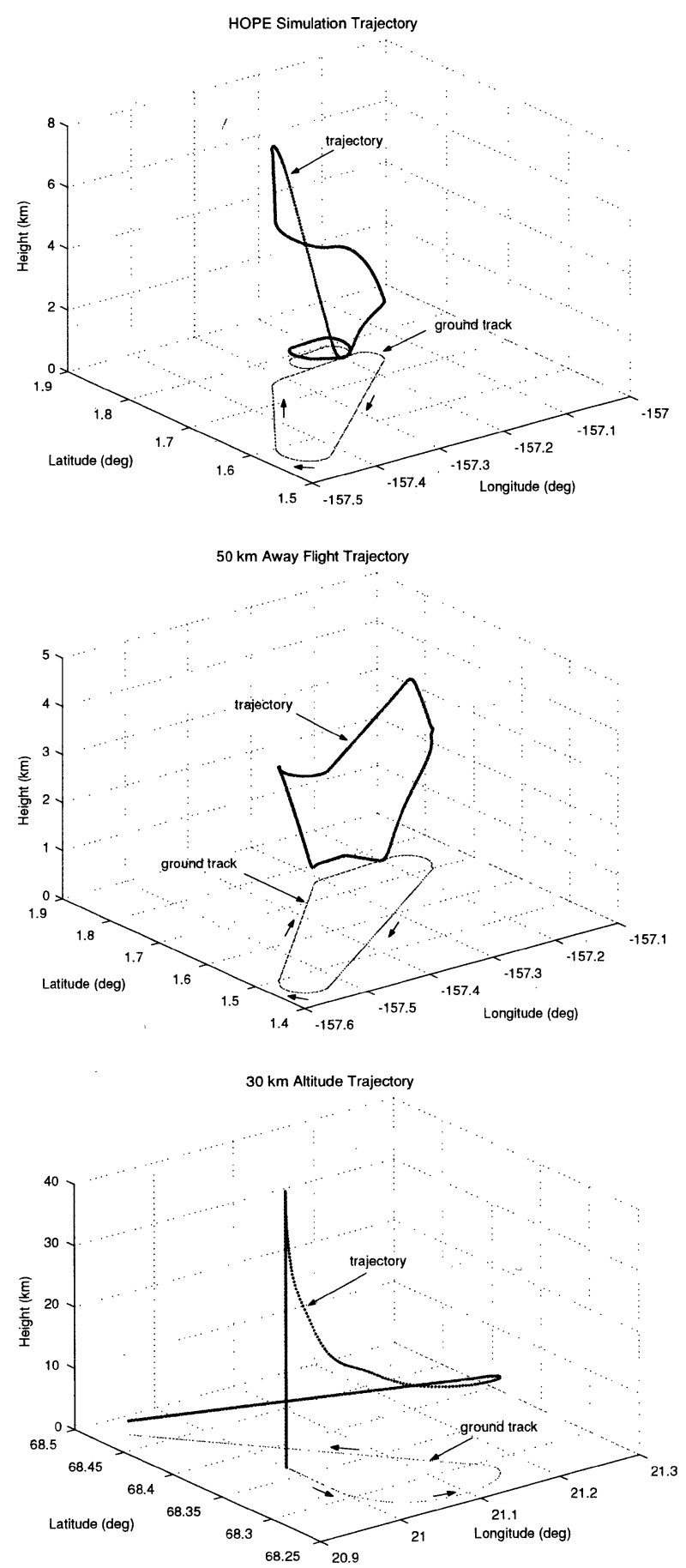

Fig. 4 HSFD trajectories used in the analysis

release time from the balloon is biggest, because visibility of GPS during the acceleration phase cannot be expected on account of the HSFD attitude and then GAINS operates in the INS mode whose accuracy is dependent on the initial condition at the release point.

Table 4 shows the results of the analysis that indicate the navigation accuracy of GAINS for the HSFD phase 1 and 2 missions at the worst cases. The result of the phase 2 shows the accuracy under the GPS/INS mode that does 
not need the telemetry link. On the other hand, the results for the phase 1 are under the CDGPS/INS mode on account of meeting the severe requirement. Because HSFD makes rapid turns with a large bank angle to fly around the runway in the HOPE simulation trajectory, the visibility of GPS becomes poor during the turn and the navigation error increases. Then there exists the period when GAINS does not meet the requirement $(1 \mathrm{~m}$, $3 \sigma$ ) for the approach phase. However, it occurs for a short period when the altitude of HSFD is still above $200 \mathrm{ft}$ (ICAO Category I decision height). And when the visibility of GPS recovers, the accuracy of GAINS improves quickly and meets the requirement below $200 \mathrm{ft}$ altitude. Then it concludes that this out of specification does not affect HSFD landing and does not lose the availability either. In other worst cases analyzed for the $50 \mathrm{~km}$ away flight trajectory and the $30 \mathrm{~km}$ altitude trajectory, the navigation accuracy satisfies the requirements for all the flight period. Therefore there is no loss of availability in the point of the accuracy of GAINS.

\section{2 Integrity and Continuity}

\subsubsection{Analysis Method}

The integrity performance means that GAINS ensures the sufficiently low probability of HMI (Hazardously Misleading Information) by its integrity monitoring function. The definition of HMI is that a Navigation System Error (NSE) exceeds some specified Alert Limit (Vertical Alert Limit (VAL) or Lateral Alert Limit (LAL)) without annunciation within the time to alarm. To simplify the analysis, we consider any excursion of NSE beyond the alert limit for any time to be hazardous. Since the true position is unknown, the actual NSE cannot be determined. But an upper bound of NSE with some specified probability can be estimated by the covariance analysis technique used in the previous section. The upper bounds of the positioning error in vertical and lateral direction are called Vertical Protection Level (VPL) and Lateral Protection Level (LPL) respectively. Therefore we say it by the following equations that GAINS satisfies the required integrity performance (to simplify the discussion, the following discussion will concentrate on the vertical direction).

$$
\begin{aligned}
& \operatorname{Pr}\left(V P L_{H 0}\right)=\operatorname{Pr}\left(V P L \mid H_{0}\right)=P_{M D_{-} F F} \\
& \operatorname{Pr}\left(V P L_{H 1}\right)=\operatorname{Pr}\left(V P L \mid H_{1}\right)=P_{M D} \\
& \operatorname{Pr}(H M I)=P_{M D_{-} F F}+P_{M D} P\left(H_{1}\right) \\
& V P L=\max \left\{V P L_{H 0}, V P L_{H 1}\right\} \leq V A L
\end{aligned}
$$

where $H_{0}$ means no fault in GAINS and $H_{1}$ means a fault on one GPS measurement. The probability of two or more measurements fault is considered to be sufficiently smaller than the integrity requirements and to be negligible. The probability of HMI $(\operatorname{Pr}(H M I))$ and the Alert Limit $(V A L)$ are defined in the integrity requirements.

The value of $V P L_{H 1}$ depends on the performance of the integrity monitoring function of GAINS that detects a GPS measurement fault by evaluating the measurement residuals at the Kalman filter. In other words, GAINS checks the quality of GPS by comparing INS every measurement update. When the following equations are satisfied, GPS measurement update is performed on a pseudorange and a carrier phase.

$$
\begin{aligned}
& \left|v_{\Delta \rho}\right|=\left|\Delta \rho-\Delta \rho_{c}\right|<T_{\Delta \rho} \\
& \left|v_{\Delta \phi}\right|=\left|\Delta \phi-\Delta \phi_{c}\right|<T_{\Delta \phi}
\end{aligned}
$$

where $\Delta \rho_{c}$ and $\Delta \phi_{c}$ are the single differences of the computed pseudorange and carrier phase respectively. We specify the thresholds $T_{\Delta \rho}$ and $T_{\Delta \phi}$ by the trade-off between the integrity performance and the continuity performance. When the threshold is smaller, the sensitivity to a GPS measurement fault is high so that Vertical Protection Level $\left(V P L_{H 1}\right)$ is small and the integrity performance improves. But a large number of correct GPS data is also rejected to update (fault-free detection) and the continuity performance degrades because of the alarm. When the threshold is larger, the opposite phenomenon is observed. To assure the continuity performance, we set the threshold dependent on the theoretical covariance of the measurement residual. The covariance of the measurement residual $\mathrm{v}$ is calculated by the following equation (same formula for the pseudorange and the carrier phase).

$$
E\left[v_{k}^{2}\right]=\boldsymbol{h}_{k}^{T} \boldsymbol{W} \boldsymbol{P}_{k}^{-} \boldsymbol{W}^{T} \boldsymbol{h}_{k}+\boldsymbol{r}_{k}
$$

Then, threshold $\boldsymbol{T}$ is denoted as follows.

$$
T=k_{F F D} \sqrt{\boldsymbol{h}_{k}^{T} \boldsymbol{W} \boldsymbol{F}_{k}^{-} \boldsymbol{W}^{T} \boldsymbol{h}_{k}+\boldsymbol{r}_{k}}
$$

where $k_{F F D}$ is the multiplier that determines the probability of fault-free detection $P_{F F D}$. GAINS is the GPS

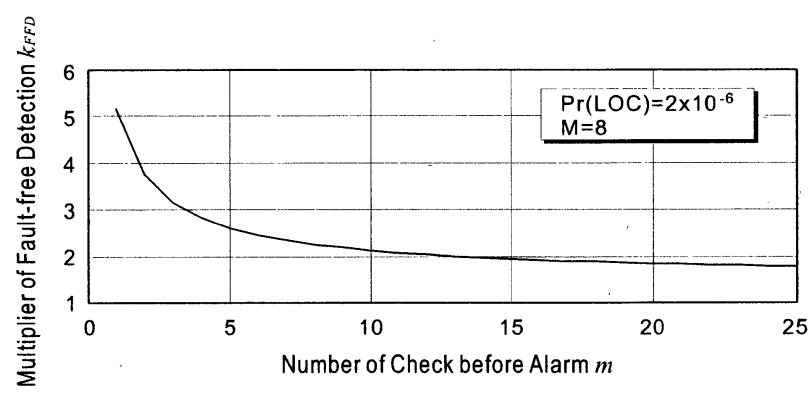

Fig. 5 Efficiency of INS Integration on Continuity 
aided inertial navigation system that is not necessary to alarm GPS fault only with one large residual. Without the measurement update, GAINS can continue to provide precise navigation data for several seconds. This feature decreases the loss of continuity (LOC) probability that is calculated as follows.

$$
\operatorname{Pr}(L O C)=\left(M \cdot P_{F F D}\right)^{m}
$$

where $M$ means the number of measurements (measurement residuals) and $m$ indicates how many epochs the measurement residuals are checked before giving the alarm. Fig. 5 shows the efficiency of $m$ on improving the performance of fault-free detection. We can get the great improvement in making the multiplier $k_{F F D}$ smaller with 3 to 5 -time checks before the alarm.

When the threshold is defined, we can calculate the probability of missed detection given one GPS measurement includes a bias error $b$ that is modeled by the equation (17).

$$
\begin{aligned}
P_{M D}(b, T)= & \int_{-\infty}^{\infty} \frac{1}{\sqrt{2 \pi} \sigma_{b}} e^{-\frac{c^{2}}{2 \sigma_{b}^{2}}} \\
& \cdot\left\{Q\left(\frac{-T-c}{\sigma_{v}}\right)-Q\left(\frac{T-c}{\sigma_{v}}\right)\right\} d c \\
b \sim & N\left(0, \sigma_{b}\right) \\
Q(x)= & \frac{1}{\sqrt{2 \pi}} \int_{x}^{\infty} e^{-\frac{\iota^{2}}{2}} d t \\
\sigma_{v}= & \sqrt{E\left[v_{k}^{2}\right]}
\end{aligned}
$$

Then Vertical Protection Level for $H_{1}$ is

$$
\begin{aligned}
V P L_{H 1} & =k_{M D} \sigma_{V}(b) \\
k_{M D} & =Q^{-1}\left(P_{M D} /\left(2 P_{M D}(b, T)\right)\right)
\end{aligned}
$$

where $\sigma_{V}(b)$ is the standard deviation of a vertical position error with the GPS measurement bias $b$. In case of no bias error, we can calculate Vertical Protection Level for $H_{0}$.

$$
\begin{aligned}
V P L_{H 0} & =k_{M D_{-} F F} \sigma_{V}(0) \\
k_{M D} & =Q^{-1}\left(P_{M D_{-} F F} / 2\right)
\end{aligned}
$$

The standard deviation of a vertical position error $\sigma_{V}$ can be estimated by the covariance analysis technique described in the previous section.

\section{3 Results of Analysis in HSFD Mission}

The HSFD missions do not determine the requirement on integrity and continuity explicitly. Then we refer LAAS specifications for Category I, II, III proposed by

\begin{tabular}{|c|c|c|}
\hline & Item & Condition \\
\hline \multirow{7}{*}{ Requirement } & \multirow[t]{5}{*}{ Integrity } & VAL \\
\hline & & $\begin{array}{l}\text { CAT I . . } 10.3 \text { m,CAT II,III } \ldots .5 .4 \mathrm{~m} \\
\operatorname{Pr}(\text { HMI })\end{array}$ \\
\hline & & $\overline{\text { CAT I } . .} 1 \times 10^{-7}$, CAT II,III $\ldots 1 \times 10^{-9}$ \\
\hline & & Time-to-Alert \\
\hline & & CAT I .. 6 sec, CATII,III $\ldots 1 \mathrm{sec}$ \\
\hline & \multirow[t]{2}{*}{ Continuity } & $\operatorname{Pr}($ LOC $)$ \\
\hline & & CAT I $\ldots 4 \times 10^{-6}$, CATII, III $2 \times 10^{-6}$ \\
\hline \multirow[b]{2}{*}{$\begin{array}{l}\text { Integrity } \\
\text { monitoring } \\
\text { function* }\end{array}$} & \multirow[t]{2}{*}{ Threshold } & Standard deviation of residuals $\sigma_{v}$ \\
\hline & & $\begin{array}{l}\text { Pseudorange } \ldots 84 \mathrm{~cm} \text {, Carrier } \ldots 27 \mathrm{~cm} \\
k_{F F I}=3.2(M=8, m=3) \\
T_{\Delta_{\rho}}=2.7 \mathrm{~m}, T_{\Delta \phi}=0.9 \mathrm{~m}\end{array}$ \\
\hline \multirow{10}{*}{ Malfunction* } & \multirow[t]{2}{*}{ Bias } & $\sigma_{b}=(1 / 3) T$ \\
\hline & & $\mathrm{P}\left(H_{1}\right)=1 \times 10^{-5}$ \\
\hline & Satellite & No down $P\left(S_{0}\right)=0.7705$ \\
\hline & \multirow{3}{*}{$P_{M I}$} & 1 GPS satellite down $P\left(S_{1}\right)=0.2295$ \\
\hline & & $P_{M I}\left(S_{0}\right)=2.2 \times 10^{-5}, k_{\Lambda I I},\left(S_{0}\right)=4.2$ \\
\hline & & $P_{\Lambda I I}\left(S_{1}\right)=7.3 \times 10^{-5}, k_{\Lambda I I)}\left(S_{1}\right)=4.0$ \\
\hline & \multirow{4}{*}{$P_{M}$ IID_FF } & $P_{M I D F F}\left(S_{0}\right)=1.1 \times 10^{-10}$ \\
\hline & & $k_{M I J)_{-} F F}\left(S_{0}\right)=6.4$ \\
\hline & & $P_{M D D_{-} F F}\left(S_{1}\right)=3.6 \times 10^{-10}$ \\
\hline & & $k_{M L \_F F}\left(S_{1}\right)=6.2$ \\
\hline
\end{tabular}
RTCA SC-159 in the analysis, which are summarized in Table 5. We use only the $50 \mathrm{~km}$ flight away trajectory because the error of GAINS converges and the accuracy is comparable to that of the HOPE simulation trajectory under Category II and III phases. In the Phase 2 mission,
HSFD does not carry out precision approach and landing and then we do not perform the integrity and continuity analysis.

Table 5 Nominal conditions supposed for the analysis

Note: * All values are for CAT II, III

Table 6 Analysis result on VPL of GAINS for HSFD phase 1 mission

\begin{tabular}{l|l|c}
\hline \multicolumn{1}{c|}{ GPS } & \multicolumn{1}{c|}{ Maximum VPL } & $\begin{array}{c}\text { Worst case } \\
\text { (averaged GDOP*) }\end{array}$ \\
\hline No down & $3.2 \mathrm{~m}$ (Fig. 7) & 09:40 take-off (4.1) \\
\hline 1 down & PRN26: $7.7 \mathrm{~m}$ (Fig. 8) & $12: 20$ take-off (8.1) \\
& PRN17: $6.8 \mathrm{~m}$ & $17: 00$ take off(10.1) \\
& PRN02: $9.9 \mathrm{~m}$ & $08: 40$ take-off(10.0) \\
& & \\
& VPL exceeds VAL & \\
& totally in 0.99\% cases. & \\
\hline
\end{tabular}

Note: * averaged for $200 \mathrm{sec}$ before full stop

According to the equation (14), we define the threshold for checking the measurement residuals. However the value of the equation is variable, which is inconvenient to be used in the onboard software. Therefore we adopt the value of the worst-case (poorest GDOP and vertical direction), which are summarized in Table 5. The number of epochs for residual check before giving the alarm is three in this analysis, which means navigation by an INS stand-alone configuration is allowable for three seconds (GPS update is done every second) even under the CDGPS/INS mode. 
To simplify the discussion, we suppose the magnitude of the bias error in the pseudorange and the carrier phase to be a third of the threshold $\left(\sigma_{b}=(1 / 3) T\right)$. In this assumption, the equation (16) shows that $96.9 \%$ GPS measurements are missed to be a fault in the GAINS integrity monitoring function and degrade the positioning accuracy. We also consider a GPS satellite down that affects GDOP in the analysis. Table 5 indicates the nominal conditions supposed in this analysis.

Table 6 summarizes the results of the analysis that show maximum VPL during the CAT II and III phases

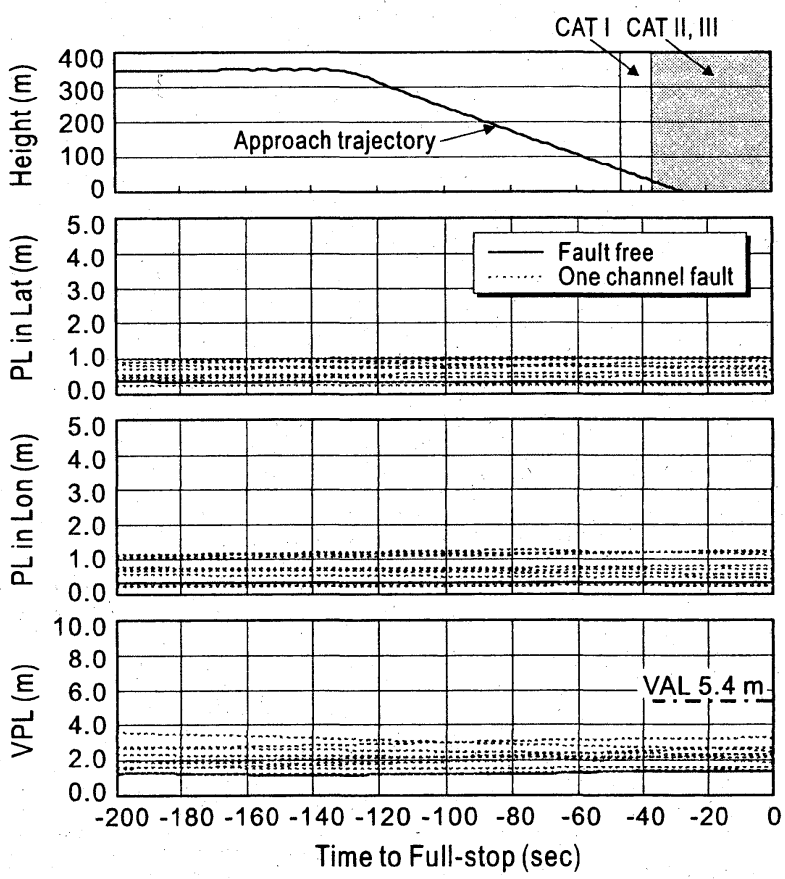

Fig. 6 VPL of no satellite down condition (Worst-case 09:40 take-off)

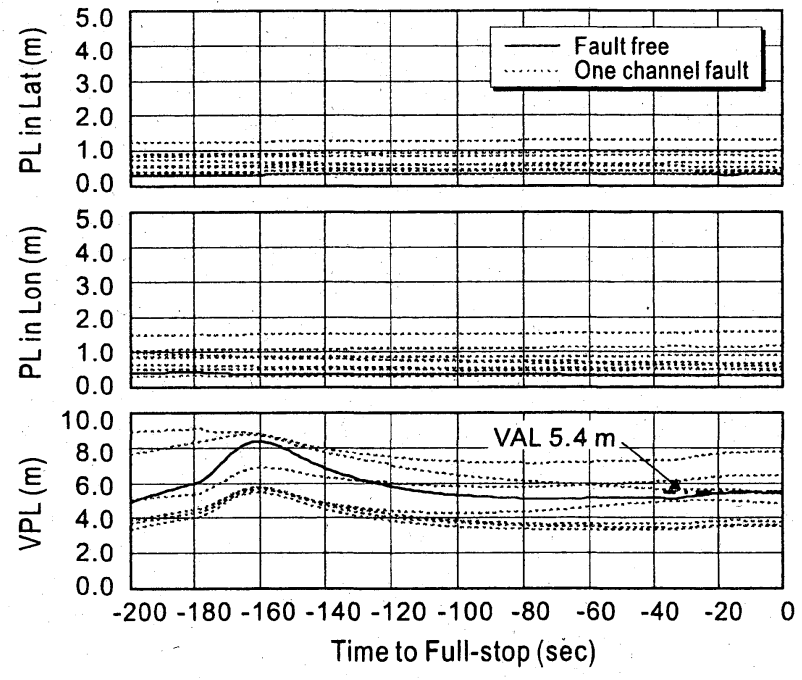

Fig. 7 VPL of PRN26 satellite down condition (Worst-case 12:20 take-off) for both no down and one down case in GPS satellites. As shown in the table, VPL of the worst case for no satellite down does not exceed VAL. Fig. 6 shows the profile of VPL in this case that plots $V P L_{H 0}$ (solid line) and $V P L_{H 1}$ (dashed line). There are plural dashed lines because a bias error of each channel has a different influence on the positioning accuracy. On the other hand, there are several cases where VPL exceeds VAL under a satellite down condition. Table 6 shows 3 cases where a satellite down causes great degrade of the integrity performance of GAINS. Fig. 7 shows VPL as an example when PRN 26 is down and HSFD takes off at 12:20 (worst case). In this case, averaged GDOP is around 8 on account of poor satellite geometry.

\section{GROUND AND FLIGHT TEST VALIDATION OF NAVIGATION PERFORMANCE}

\section{1 GAINS Flight Model}

Fig. 8 shows photos of the GAINS flight model. Fig. 8a a shows the onboard equipment that consists of a GPS/IMU/Processor package (HNU: Hybrid Navigation Unit) and a telemetry receiver. HNU uses Ashtech G12 as a GPS receiver, Kearfott T24 as an IMU and 486DX4 (66 MHz clock) as a navigation processor. Fig. $8 \mathrm{~b}$ presents the ground station that is divided into a DGPS reference system and a monitor system. Table 7 indicates the specifications of $\mathrm{HNU}$.

\section{2 TEST RESULTS}

We do VPL check every 20 minutes for a take-off window $(6: 00-18: 00)$ and find that $0.99 \%$ of total cases do not meet the integrity requirement. It means the availability is lost for $0.23 \%$ cases because the probability of one GPS satellite down is 0.2295 . Therefore the availability of GAINS for HSFD missions is 0.998, which meets the availability requirement of HSFD.

We performed various tests on the ground and in the air to verify the performance of GAINS. In this section, we concentrate on the positioning accuracy confirmed by the experiments. Table 8 summarizes the results to demonstrate the GAINS positioning accuracy on the ground and in the air. We consider that the ground tests are to verify the long-term accuracy and the repeatability. Fig. 9 shows the positioning error of GAINS under the CDGPS/INS mode when we do a 12-hour heat run test on the ground. It shows that the vertical positioning error is bounded within $0.83 \mathrm{~m}$ (mean $=0.14 \mathrm{~m}, 3 \sigma=0.69 \mathrm{~m}$ ). The accuracy of the INS mode in the ground test is derived from 21-time test data to confirm the repeatability 
Table 7 Specifications of GAINS onboard equipment

\begin{tabular}{|c|c|c|}
\hline & Item & Spec. \\
\hline \multirow{13}{*}{$\mathrm{HNU}$} & Size & $\mathrm{H} 180 \mathrm{~mm} \times \mathrm{W} 180 \mathrm{~mm} \times \mathrm{D} 280 \mathrm{~mm}$ \\
\hline & Weight & $10 \mathrm{~kg}$ \\
\hline & Power & $46 \mathrm{~W}(28 \mathrm{VDC})$ \\
\hline & Functions & $\begin{array}{l}\text { CDGPS/INS,DGPS/INS, GPS/INS, } \\
\text { INS }\end{array}$ \\
\hline & Environment & Temperature: $-40 \sim+55$ degree Celsius \\
\hline & & Altitude: $0 \sim 32 \mathrm{~km}$ \\
\hline & & Humidity: $<85 \%$ \\
\hline & & Acceleration: $8 \mathrm{G}$ \\
\hline & & Shock: 15G (11 msec) \\
\hline & & $\begin{array}{l}\text { Vibration: } \quad 0.04 \mathrm{G}^{2} / \mathrm{Hz} \quad(15 \sim 1000 \\
\mathrm{Hz})\end{array}$ \\
\hline & Interface & MIL-STD-1553B \\
\hline & Data rate & $50 \mathrm{~Hz}$ \\
\hline & Reliability & MTBF: more than 3,700 hour \\
\hline
\end{tabular}

Table 8 Test result on positioning accuracy of GAINS

\begin{tabular}{|c|c|c|}
\hline & Mode & Test result \\
\hline \multirow{4}{*}{ Ground test } & CDGPS/INS (a 12hr & Lat. $0.34 \mathrm{~m}$ (max) \\
\hline & heat run test) & Lon. $0.29 \mathrm{~m}(\max )$ \\
\hline & & $\mathrm{V} 0.83 \mathrm{~m}(\max )$ \\
\hline & INS (21 static tests) & 0.37 - 2.20 n.m.@1 hr \\
\hline \multirow{6}{*}{ Flight test } & CDGPS/INS (12 & (1nectan: $48 \mathrm{max})$ \\
\hline & approach cases) & V $0.62 \mathrm{~m}(\max )$ \\
\hline & GPS/INS (3 cases) & Lat. $3.7 \mathrm{~m}$ (max) \\
\hline & & Lon. $1.9 \mathrm{~m}(\max )$ \\
\hline & & V $5.9 \mathrm{~m}(\max )$ \\
\hline & INS ( 2 cases) & $0.59 \sim 0.85$ n.m.@1 hr \\
\hline
\end{tabular}

Note: L $\cdots$ Lateral, V $\cdots$ Vertical

Position error of the INS mode is evaluated in Horizontal.

On the other hand, the flight experiments authorize the GAINS a.ccuracy under HSFD similar conditions such as suffering GPS lock-loss and telemetry lock-loss for a short period on account of maneuvers. We performed 7 flights where we check the positioning accuracy of the

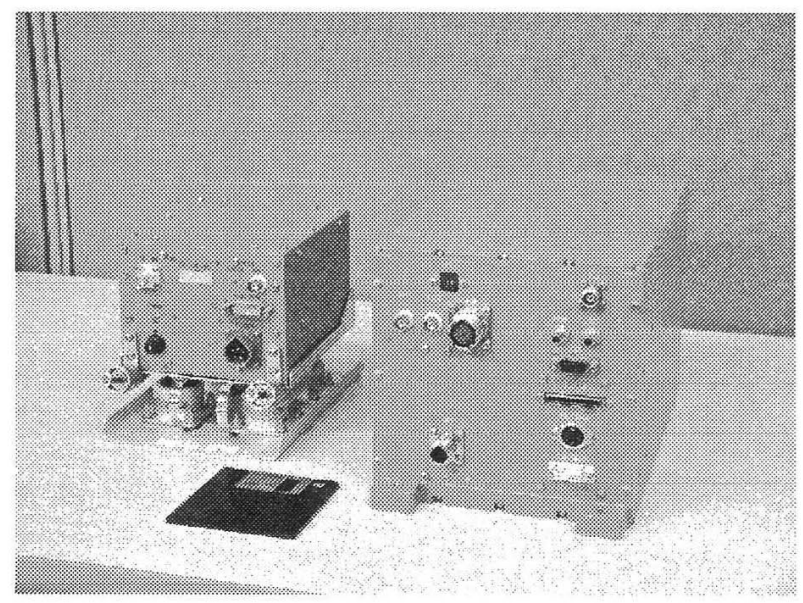

Fig. 8a GAINS onboard equipment (Right: HNU, Left: Telemetry receiver)

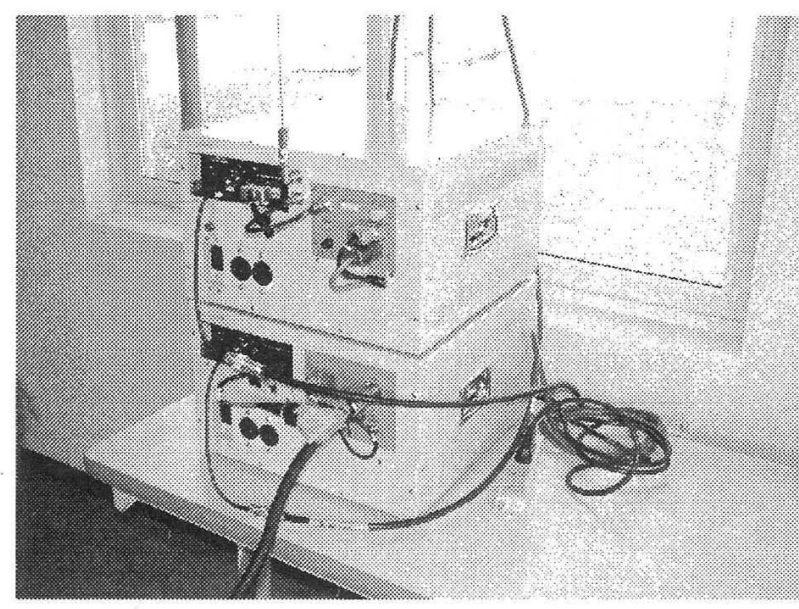

Fig. 8b GAINS ground station (Upper: Monitor system, Lower: DGPS Reference system)

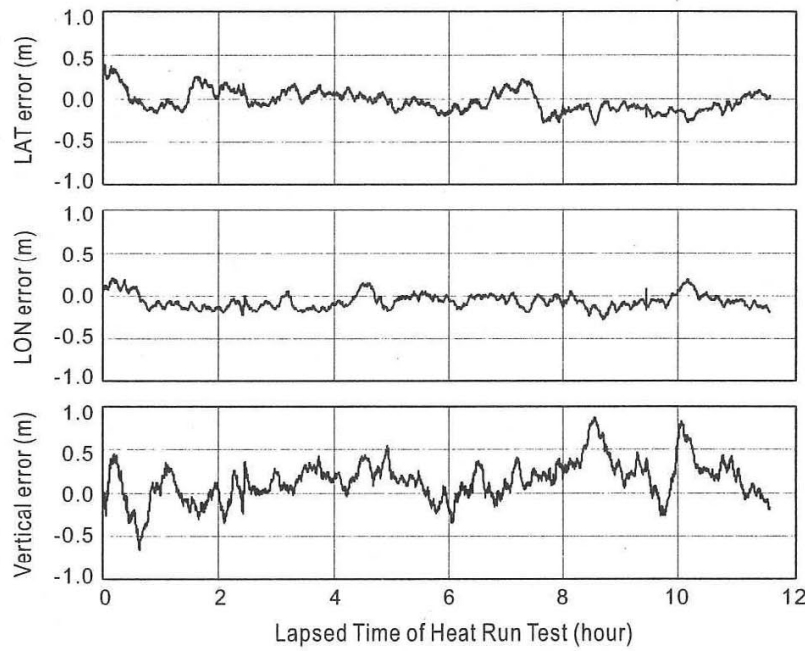

Fig. 9 Heat run test result of GAINS under CDGPS/INS mode

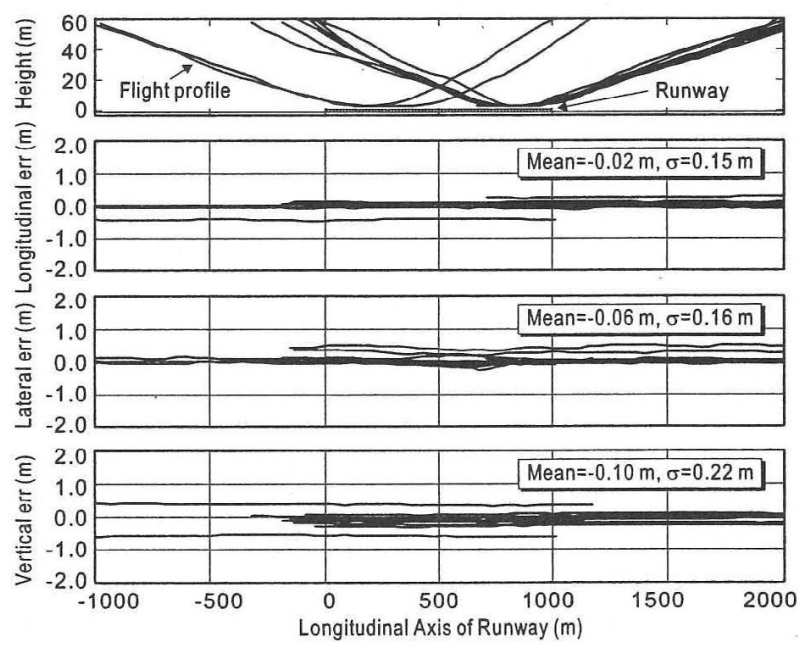

Fig. 10 Accuracy of GAINS during approach and landing under CDGPS/INS mode

CDGPS/INS, GPS/INS and INS mode. During the flight experiment, we carried out 12 approach and landing (in- 
cluding touch and go) under the CDGPS/INS mode, whose results are shown in Fig. 10. In all cases, the GAINS accuracy does not exceed the requirement ( $1 \mathrm{~m}$ $(3 \sigma))$. And short-period GPS lock-loss does not affect the positioning accuracy either as we previously estimate. The accuracy of the CDGPS/INS in the flight conditions is comparable to that of the ground tests and the theoretical analysis. It means that the flight experiments confirm the design of GAINS in HSFD conditions whose distinctive feature is CDGPS/INS hybrid navigation. The accuracy of the GPS/INS and INS mode also meet the requirements. And both performances are also rational in the point of the theoretical analysis and the sensor specifications. The transition among the three modes is also well done. We also confirm the transition philosophy that the most accurate mode available under the GPS and telemetry conditions is carried out.

\section{CONCLUSIONS}

We show the following conclusions that are derived from the theoretical analysis and test results presented in this paper.

We propose the novel approach to use the carrier phase measurement in the GPS aided inertial navigation system. The CDGPS/INS hybrid navigation method is appropriate to improve the positioning accuracy without loss of good continuity even under the HSFD severe dynamics.

We propose the analysis method of estimating the navigation accuracy of CDGPS/INS and GPS/INS hybrid navigation, which uses the covariance analysis technique The results of analysis assure that GAINS meet the requirements of IISFD missions whose most severe requirement exists in vertical position error under the approach and landing phase.

We propose the analysis method of evaluating the integrity and continuity performance of CDGPS/INS hybrid navigation. The INS integration is useful to ease the continuity requirement and the measurement residual check is efficient to reduce VPL. The results show the availability of GAINS in the HSFD missions is 0.998 . The main reason to degrade the availability is the GPS satellite failure that causes the poor satellite geometry.

We construct the GAINS fight model. By the ground and flight tests, we verify the design of CDGPS/INS hybrid navigation. The accuracy under the approach and landing phase satisfies the requirement of the HSFD missions and confirms the correctness of the theoretical analysis.

GAINS shows highest-level performance among the de- veloped GPS/INS systems. Its technology may be useful for manned general aviation that requires high reliability and tolerance to unintentional interferences.

\section{References}

1) T. Nishizawa, M. Sagisaka, H. Inokuchi and M. Harigae: Avionics Architecture for HOPE-X High Speed Flight Demonstrator, Proceedings of AIAA/NAL-NASDA-ISAS 10th International Space Planes and Hypersonic Systems and Technologies Conference, AIAA-2001-1835 (2001)

2) M. Harigae, et.al: Development of Precision Approach and Landing Navigation System Using the Carrier-phase DGPS/INS Hybrid Navigation Algorithm, Technical Paper of National Aerospace Laboratory, TR-1399 (2000)

3) RTCA Working Group SC-159: Minimum Aviation Performance Standards for the Local Area Augmentation System (LAAS), Document No. RTCA/DO-245 (1998)

4) F. Liu, T. Murphy and T. Skidmore: LAAS Signal-inSpace Integrity Monitoring Description and Verification Plan, Proceedings of the 10th International Technical Meeting of the Satellite Division of the Institute of Navigation, ION GPS-97, 485/497 (1997)

5) M. Harigae, T. Tsujii, T. Ono, T. Inagaki and H. Tomita: Flight Evaluation of Carrier DGPS/INS Hybrid Navigation for Automatic Landing, Proceedings of the 11th International Technical Meeting of the Satellite Division of the Institute of Navigation, ION GPS-98, 437/446 (1998)

6) R. Hatch: Instantaneous Ambiguity Resolution, Proceedings of IAG International Symposium No.107 on Kinematic Systems in Geodesy, Surveying and Remote Sensing, New York: Springer Verlag, 299/308 (1991)

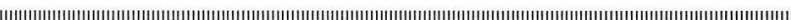
Masatoshi Harigae (Member)

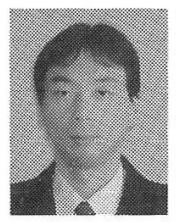

- Masatoshi Harigae is the group leader of Navigation Systems Group of Flight Systems Research Center in NAL, who has over 15 years experience in the development of a. GPS/INS hybrid navigation system. He has a B.S., a M.S. and a. PhD from the University of Tokyo in aerospace engineering.

\section{Hiroshi Tomita}

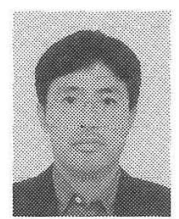

Hiroshi Tomita is a senior researcher of Flight Experiment Group of Flight Systems Research Center in NAL. He has over 10 years experience in the development, of GPS applied navigation system, both for space and aeronautics. He has a M.S in engineering.

\section{Takeshi Nishizawa}

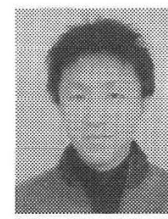

Takeshi Nishizawa is a member of HOPE-X project team in NASDA, who has been engaged in development of a HOPE-X avionics system. He has a B.Eng., a M.Eng. from the University of Hokkaido in physical science and engineering. 